\title{
PROMOÇÃO DA SAÚDE NO CONTEXTO PAROARA: POSSIBILIDADE DE CUIDADO DE ENFERMAGEM
}

\author{
Regina Ribeiro Cunha1, Luciléia da Silva Pereira², Ana Sofia Resque Gonçalves', Evanguelia Kotzias Atherino \\ dos Santos ${ }^{4}$ Vera Radünz ${ }^{5}$ Ivonete Teresinha Schülter Buss Heidemann ${ }^{6}$
}

\footnotetext{
${ }^{1}$ Doutoranda do Programa de Pós-Graduação da Universidade Federal de Santa Catarina (PEN/UFSC). Professora Assistente do Departamento de Enfermagem da Universidade do Estado do Pará (UEPA) e da Faculdade de Enfermagem da Universidade Federal do Pará (UFPA). Bolsista da Coordenação de Aperfeiçoamento de Pessoal de Nível Superior (CAPES). Pará, Brasil. E-mail: oirainha@gmail.com

${ }^{2}$ Doutoranda do PEN/UFSC. Professora Assistente da Faculdade de Enfermagem da UFPA. Bolsista da CAPES. Pará, Brasil. E-mail: lucileiapereira21@yahoo.com.br

${ }^{3}$ Doutoranda do PEN/UFSC. Professor Adjunto da Faculdade de Enfermagem da UFPA. Bolsista da CAPES. Pará, Brasil. E-mail: sofiares@ufpa.br

${ }^{4}$ Doutora em Enfermagem. Professor Associado do Departamento de Enfermagem e do PEN/UFSC. Santa Catarina, Brasil. E-mail: gregos@matriz.com.br

${ }^{5}$ Doutora em Enfermagem. Professor Associado do Departamento de Enfermagem e do PEN/UFSC. Santa Catarina, Brasil. E-mail: radunz@nfr.ufsc.br

${ }^{6}$ Doutora em Saúde Pública. Professor Adjunto do Departamento de Enfermagem e do PEN/UFSC. Santa Catarina, Brasil. E-mail: ivonete@ccs.ufsc.br
}

\begin{abstract}
RESUMO: O cuidado é considerado um bem social imensurável cujo significado está atrelado à vida e constitui uma das ações que visa a manutenção e promoção da saúde. O enfermeiro, como profissional ativo e transformador do ambiente social, tem o cuidado como elemento essencial de sua práxis. Esta reflexão tem como objetivo discutir uma das estratégias de promoção da saúde, o desenvolvimento de habilidades/atitudes pessoais, como possibilidade de cuidado de enfermagem para a melhoria da saúde dos paroaras. Pontua aspectos contextuais do cuidado, com ênfase na educação. Parte do princípio das atitudes pessoais da promoção, apontando desafios antropológicos no contexto sociocultural da Amazônia. Evidencia a necessidade de desenvolver habilidades pessoais como uma das estratégias que pode contribuir para o cuidado individual e coletivo, e o princípio do reforço da ação comunitária, essencial para o cuidado de enfermagem dos paroaras.
\end{abstract}

DESCRITORES: Promoção da saúde. Enfermagem. Enfermagem transcultural. Educação em saúde.

\section{HEALTH PROMOTION IN THE PAROARA CONTEXT: THE POSSIBILITY FOR NURSING CARE}

\begin{abstract}
Care is considered an immeasurable social benefit whose meaning is tied to life itself. It constitutes an action that seeks the maintenance and promotion of health. The nurse, as an active professional and social environment transformer, includes care as an essential element of professional practice. The objective of this reflection is to discuss one health promotion strategy - the development of personal abilities/attitudes - as a possibility for nursing care in improving the health of paroaras. It points out contextual aspects of care, focusing on education. It adopts the personal behaviors towards promotion as a starting point, highlighting anthropological challenges in the socio-cultural context of the Amazon. It evidences the need to develop personal skills as one of strategies that can contribute to individual and collective care and the principle of reinforcing community action, an essential element for nursing care for paroaras.

DESCRIPTORS: Health promotion. Nursing. Transcultural nursing. Health education.

\section{LA PROMOCIÓN DE LA SALUD EN EL CONTEXTO PAROARA: LA POSIBILIDAD DE ATENCIÓN DE ENFERMERIA}

\begin{abstract}
RESUMEN: El cuidado es considerado un bien social inmensurable cuyo significado está vinculado a la vida. Constituye una de las acciones que busca la manutención y promoción de la salud. El enfermero como profesional activo y transformador del ambiente social, tiene al cuidado como un elemento esencial de su práxis. El objetivo de esta reflexión es discutir una de las estrategias de promoción de la salud: el desarrollo de las habilidades y actitudes personales como posibilidades de cuidado para la mejoría de la salud de los paroaras. El estudio señala aspectos contextuales del cuidado, con énfasis en la educación. Parte del principio de las actitudes personales de la promoción, señalando desafíos antropológicos en el contexto socio-cultural de la Amazonia. Muestra la necesidad de desarrollar habilidades personales como una de las estrategias que pueden contribuir para el cuidado individual y colectivo, y el principio del refuerzo de la acción comunitaria como fundamental para la atención de enfermería de los paroaras.
\end{abstract}

DESCRIPTORES: Promoción de la salud. Enfermería. Enfermería transcultural. Educación en salud. 


\section{CONSIDERAÇÕES INICIAIS}

Estudos sobre a promoção da saúde têm despertado interesse de diversos autores na produção do conhecimento da enfermagem. A definição de promoção da saúde apresentava uma variedade de teorias, conceitos de estratégias de intervenção, até a divulgação da Carta de Otawa em 1986, Canadá. A partir desta, foi definida como: "o processo que capacita as pessoas e indivíduos para atuarem na melhoria da qualidade de vida e saúde, incluindo uma maior participação no controle desse processo". 1:1

Nessa perspectiva, a promoção da saúde passa a ser incorporada de forma mais ampla e abrangente não se restringindo ao enfoque estritamente biologicista. Vislumbra-se uma mudança de paradigma desde o modelo de Leavell e Clark, no qual a promoção da saúde constituía níveis de atenção primária à saúde. ${ }^{2}$ Esta concepção se refere a uma combinação de estratégias: políticas públicas saudáveis, criação de ambientes saudáveis, reforço da ação comunitária, desenvolvimento de habilidades pessoais, reorientação do sistema de saúde. ${ }^{3}$

A dificuldade em seguir as cinco principais estratégias de promoção da saúde implica na mudança do estilo de vida das pessoas, das atividades laborais e do lazer, tendo um expressivo impacto sobre a saúde. ${ }^{4}$

Neste ensaio, focaliza-se uma das estratégias prioritárias de promoção da saúde a saber: o desenvolvimento de habilidades pessoais, em que se busca capacitar as pessoas para "aprender através da vida" e prepará-las para todos os estágios. ${ }^{5}$ Traduz-se como uma possibilidade das pessoas organizarem seu próprio cuidado com a saúde, ancoradas em suas experiências vivenciadas.

Sob este prisma, a promoção da saúde envolve múltiplas dimensões para o desenvolvimento da autonomia das pessoas e não pode ser realizada com base em uma única área do conhecimento. A consolidação de outras ciências e saberes, além da racionalidade biomédica, resulta de uma superação do individualismo rumo ao âmbito coletivo, institucional e estrutural. ${ }^{6}$

No bojo dessa discussão, vale salientar que a consolidação do saber da Enfermagem teve origem com a necessidade de cuidado. ${ }^{7} \mathrm{O}$ cuidado constitui uma das ações essenciais (e na atualidade mais do que nunca) que visa a manutenção da vida. Assim, a promoção da saúde é apontada como aprimoramento das habilidades vitais para o cuidado, logo é essencial e deve ser considerada como uma possibilidade de cuidado, contribuindo para a melhoria da qualidade de vida, bem como para a saúde dos paroaras.

A presente reflexão é uma tentativa exploratória inicial, que contempla a promoção da saúde no contexto paroara como possibilidade de cuidado de enfermagem, tendo como objetivo refletir sobre uma das estratégias de promoção da saúde, o desenvolvimento de habilidades/ atitudes pessoais como possibilidade de cuidado de Enfermagem para a melhoria da saúde dos paroaras. Em termos metodológicos, o desenho do presente estudo se caracteriza como uma reflexão teórica acerca da temática que propomos discutir. Seguimos o percurso cuidado de enfermagem e promoção da saúde e, perspectiva antropológica do cuidado de enfermagem.

\section{CUIDADO DE ENFERMAGEM E PROMO- ÇÃO DA SAÚDE}

A jovem Florence Nightingale, ao cuidar de feridos na Guerra da Criméia, no ano de 1854, exerceu sua prática com olhar holístico considerando a influência do ambiente nas reações humanas das vítimas da guerra. ${ }^{8}$ Sua atitude foi muito empreendedora para a época. Nightingale deixou um legado de persistência, capacidade, compaixão e dedicação ao próximo. Provavelmente não imaginava que seu nome entraria definitivamente na história, pois estabeleceu diretrizes e o caminho para Enfermagem moderna.

Nessa perspectiva, cabe ressaltar que o cuidado em enfermagem, contém em sua essência um caráter humanístico. ${ }^{9}$ Ao incorporar o contexto da liberdade e da autonomia, individual e coletiva em diversas sociedades humanas, o cuidado de enfermagem constitui-se como um "[...] suporte para viver bem, promovendo condições para uma vida saudável e em benefício do bem comum" . 9:268

Estudo atual revelou que a Enfermagem é constituída por um "[...] modo especial de diálogo humano" ${ }^{10: 547}$ Nesse sentido, o cuidado de enfermagem pode, então ser entendido como uma relação entre as pessoas que pretendem transformar-se por meio de suas relações de cuidar, com vistas a promoção, manutenção ou recuperação da saúde. ${ }^{10}$

O cuidado de enfermagem não requer somente aplicação para o bem-estar físico e mental

* Refere-se às pessoas originárias do Estado do Pará - Brasil.

Texto Contexto Enferm, Florianópolis, 2009 Jan-Mar; 18(1): 170-6. 
das pessoas. É preciso valorizar e agir a partir de "efetivos rearranjos tecnológicos", com ênfase nas interações dialógicas, por meio da exploração de linguagens diferenciadas, como a linguagem e a expressão artística, valorizando os aspectos socioculturais do processo saúde-doença.

Nessa perspectiva, a promoção da saúde incorpora mais efetivamente o cuidado de enfermagem, transcendendo o enfoque estritamente biológico. Sabe-se que o cuidado é inato, é inerente, é próprio de todo ser vivo. Animais irracionais demonstram ações de cuidado com suas crias, acolhendo-as, provendo-as de alimento e de abrigo e instruindo-as para sua independência. $\mathrm{O}$ ser humano expressa a magnitude de sua capacidade de cuidado desde a concepção até a morte, quando os mortos são acomodados em caixões acolchoados de cetim resgata-se o conforto de uma cama para repousar. ${ }^{11}$ Essa conduta traduz uma ação de cuidado.

O cuidado, como bem social imensurável atrelado à vida cotidiana das pessoas, em qualquer sociedade, requer habilidades/atitudes específicas, inerentes ao contexto no qual está inserido o sujeito envolvido nessa ação. Nesse sentido, a saúde é pensada como a meta de todo cuidado. ${ }^{12}$ O cuidado sob a ótica da promoção da saúde pode ser visto como uma das possibilidades a ser focalizada pela Enfermagem, desde que voltada para o conceito ampliado do processo saúde-doença. Assim, é na práxis da Enfermagem que o cuidado se efetiva. A Enfermagem pode contribuir ou comprometer-se com a promoção da saúde, seja individual ou coletiva, por meio do cuidado com uma lente apropriada para olhar e cuidar, considerando a diversidade cultural.

Portanto, o cuidado com a saúde pode ser priorizado e organizado de acordo com diferentes concepções do processo saúde-doença sobre o desenvolvimento humano e, de acordo com o contexto sociocultural. Neste sentido, o cuidado na sociedade se reveste de um simbolismo que tem uma multidimensionalidade cultural em todos os lugares.

Compreende-se, assim, que todas as ações do enfermeiro na produção de assistência deveriam se efetivar por meio do cuidado, da educação, informação, comunicação e do gerenciamento, tendo a finalidade de atender necessidades da população relacionadas à manutenção da saúde como condição de sua natureza de ser vivo. Nesse sentido, a promoção da saúde pode ser considerada uma possibilidade de cuidado.
Um componente que consideramos relevante no âmbito da promoção da saúde é a educação, que consiste em um processo de eleição, assimilação e transformação de valores recebidos e que passam de geração a geração e à criação de outros novos valores. ${ }^{13} \mathrm{O}$ tema da educação em saúde se insere no contexto da promoção da saúde, quando se trabalha com a estratégia de ação do desenvolvimento das habilidades pessoais, entendida por Freire como a ação que pode e deve ser muito mais do que um processo de treinamento ou domesticação; um processo que nasce da observação e da reflexão e culmina na ação transformadora. ${ }^{14}$

A educação está fundamentada na reflexão a partir da realidade do educando, o qual após retornar a essa realidade tem a possibilidade de transformá-la. ${ }^{14}$ Para o educador, o ser humano estabelece relações com a natureza e a cultura. Parafraseando o pensamento freireano, o enfermeiro pode ser considerado, juntamente com o indivíduo, a família e a comunidade, também um educando.

Ao conceber o cuidado como atividade de educação em saúde, a Enfermagem deixa de se sentir a "dona" do cuidado, e adotando uma postura horizontalizada. Exercendo esse cuidado holístico, contribui para a construção de uma prática crítica, libertadora e contextualizada, valorizando a culturalidade do indivíduo, família e comunidade. ${ }^{14}$

Essa perspectiva é contemplada no perfil do egresso/profissional do Curso de Graduação em Enfermagem da Universidade Federal do Pará, uma vez que o mesmo deve ser "[...] Capaz de conhecer e intervir sobre os problemas/situações de saúde-doença mais prevalentes no perfil epidemiológico nacional, com ênfase na região Amazônica, identificando as dimensões bio-psico-sociais dos seus determinantes. Capacitado a atuar, com senso de responsabilidade social e compromisso com a cidadania, como promotor da saúde integral do ser humano". ${ }^{15: 4}$

Embora esse projeto político aponte para tendências de um ensaio de transdisciplinaridade, o enfermeiro ainda não despertou para o papel social que exerce como agente transformador da realidade. Isso demonstra que há uma deficiente articulação dos conteúdos filosóficos, sóciopolítico, histórico e antropológico, que dificulta ao profissional de enfermagem ter visão crítica da realidade social e o seu papel político de agente transformador. Vale ressaltar que o enfermeiro tem, sob sua coordenação, o maior contingente quantitativo e qualitativo de profissionais de 
enfermagem que, atuando junto ao indivíduo, família e à comunidade por mais tempo, poderia tornar-se um mediador do sistema de saúde capaz de incorporar formas alternativas de promover a saúde dos paroaras.

O papel da educação numa perspectiva libertadora é proporcionar o verdadeiro diálogo entre os profissionais de saúde e a população. O profissional que tem o compromisso ético, libertador, progressista na sua prática cotidiana deve desafiar a população a "pensar criticamente a realidade social, política e histórica em que é uma presença". ${ }^{14: 44} \mathrm{Na}$ realidade, a promoção da saúde pode contribuir com esta diversidade cultural, ampliando o atendimento das capacidades humana, melhorando a qualidade de vida e propiciando maior emancipação das capacidades individuais e coletivas.

Os indivíduos e os grupos devem saber identificar suas aspirações, satisfazer necessidades e modificar favoravelmente o meio ambiente. A saúde, desta forma, deve ser vista como um recurso para a vida que enfatiza os recursos sociais e pessoais, bem como as capacidades físicas. Assim, a promoção de saúde não é responsabilidade exclusiva do setor saúde, indo além das abordagens de estilos de vida saudáveis em direção de um bem-estar global. Partindo-se desse pressuposto, a promoção da saúde implica em definição de ações educativas para o desenvolvimento das habilidades da população na busca por caminhos que apontem para um viver saudável.

Paralelamente, o enfrentamento dos determinantes de saúde em toda a sua amplitude requer uma efetiva articulação intersetorial do poder público e da mobilização da população. Para tanto é necessário resgatar a participação popular, que tem sido vista como um conceito importante desde a Declaração de Alma Ata ${ }^{16}$ e reforçada por meio da idéia de emancipação defendida pela Carta de Ottawa. ${ }^{1}$

\section{A PERSPECTIVA ANTROPOLÓGICA DO CUIDADO DE ENFERMAGEM}

Conceitos e métodos da Antropologia podem nos ajudar a compreender mais ricamente os sujeitos com os quais interagimos nas e pelas ações de saúde. A palavra Antropologia deriva de dois vocábulos gregos: anthropos (homem) e logos (estudo), exprimindo a idéia de "estudo do homem". Essa ciência tenta proporcionar uma visão integrada do "problema da existência humana". 13:30 "A utilização da Antropologia e de seu método para abordar e ilustrar as situações de cuidado, permite captar como se estrutura uma situação sociocultural e, extrair laços de significação que devem decodificar-se para que os cuidados sejam apropriados". 17:156

A compreensão cultural de um grupo resulta da interpretação do comportamento dos sujeitos que o compõe. Na interpretação, buscam-se os significados das ações da vida das pessoas em uma determinada sociedade, as quais se entrelaçam, transformando-se em visões de mundo, relacionados a determinadas formas de ethos e comportamentos. ${ }^{18}$ Nessa perspectiva, entende-se que os seres humanos vivem em diferentes lugares e contextos.

Neste sentido, é importante considerar que os paroaras habitam a região Amazônica, a mais densa bacia hidrográfica do globo, cuja população tem nos rios sua mais concreta e viável estrada natural, como também, com grande representatividade, a base onde estão suspensas suas residências. Enfrentam uma situação paradoxal, pois, é uma região rica em recursos naturais, que ao longo de sua história tem sido explorada por um sistema que privilegia uma minoria detentora do capital, e que tem, no entanto, parte de seu contingente populacional empobrecida e, conseqüentemente, sem condições satisfatórias de saúde. ${ }^{19}$

O grande fluxo migratório no Estado do Pará, porta de entrada para cidadãos menos favorecidos da região nordeste do Brasil e também de parte da região centro-oeste, tem aumentado o índice populacional de pessoas com menor poder aquisitivo, intensificando o número de pessoas abaixo da linha de pobreza. Este padrão de vida mínimo vem contribuindo para o aumento de todas as demandas sociais, econômicas, políticas e culturais da população local.

Grande parte da população ainda vive da agricultura de subsistência e em precárias condições de educação e saúde, situação que demanda ações de promoção da saúde para o cuidado e melhoria da qualidade de vida, respeitando as características regionais, pois, embora tenha contato com pessoas de outras regiões, o povo da Amazônia ainda conserva peculiaridades culturais que podem ser consideradas como distintas.

Essas peculiaridades associadas às desigualdades sociais fazem dessa região um desafio que se sobrepõe a qualquer outro referente à manutenção da população brasileira. Dessa forma, o cuidado de enfermagem na região Amazônica, especificamente no Pará, significa criar estratégias que possibilitem o desenvolvimento de habilidades pessoais a esta população que vive em condições diferenciadas. 
Essas condições nos remetem aos dados publicados pelo governo do Estado, os quais mostram que, na região metropolitana de Belém, se concentram $29,30 \%$ da população do Estado. Em 2005, tinha 781.021 pessoas pobres, com um incremento de 14,69\% em relação a 2004. Quando se compara com o total da população estadual, essa população abaixo da linha de pobreza correspondia a $49,40 \%$ em 2005 , quase a metade da população do Estado. ${ }^{20}$ Dessa forma, provavelmente apenas com o crescimento econômico isolado não será possível eliminar os problemas sociais. As políticas públicas devem favorecer o crescimento econômico, priorizando a desconcentração de renda com vistas à redução efetiva do número de pessoas abaixo da linha de pobreza.

Nos diferentes contextos da região Amazônica emergem possibilidades de potencializar a promoção da saúde incorporada ao saber popular. Nesta região, a herança cultural de sua população está imersa em raízes da cultura indígena, atribuindo à pajelança uma possibilidade capaz de buscar alternativas para solução de problemas que possam interferir no viver saudável. Para isso, torna-se necessário que a Enfermagem desenvolva um olhar cuidativo à saúde em detrimento da doença, considerando a diversidade cultural.

Pesquisadores da Antropologia se ocupam em estudar as concepções de saúde-doença, valorizando a diversidade cultural. ${ }^{21-23}$ Ao contrário do que ocorre no caso da medicina ocidental, forjada dentro da tradição individualizante, a pajelança, como outras medicinas populares da Amazônia e de outras partes do mundo, assume, por meio dos métodos de tratamento do pajé, um caráter holístico, totalizante, que também é condizente com a ideologia dos sujeitos populares que a procuram para tratar-se de seus males físicos e tentar resolver seus conflitos psíquicos, assim como seus problemas nas relações interpessoais.22

Nessa linha de pensamento, a Enfermagem precisa identificar e compreender as diversas dimensões do cuidado em distintos contextos culturais. Assim, essa ciência com seu saber e recursos especializados passa a ser mediadora do encontro entre a população e o conjunto de recursos de que uma sociedade dispõe (informações, serviços, insumos e outros) para promover sua saúde. ${ }^{24}$

Nesse contexto, Madeleine Leininger, ${ }^{25}$ teórica da Enfermagem, destaca-se, ao criar a Teoria da Diversidade e Universalidade Cultural de Cuidado, cuja premissa define que o cuidado de enfermagem emerge no próprio contexto cultural onde daí se desenvolve. ${ }^{26}$ Leininger, na década de 50, ao desenvolver atividades com um grupo de crianças com problemas mentais de diferentes nacionalidades, percebeu que elas demandavam maneiras de cuidar diferenciadas. ${ }^{26-27}$

As concepções de cuidar, com base em publicações de enfermeiras americanas e brasileiras, apontam as várias perspectivas no âmbito cultural, organizacional, estético, existencialista e consciencial. Na perspectiva cultural, destaca-se, dentre as considerações propostas por Leininger, a idéia de que é essencial que a Enfermagem como profissão e disciplina global e universal possa identificar o que é universal e o que é peculiar no cuidado humano e no cuidado profissional de enfermagem, considerando as multiculturas. ${ }^{28}$

Desse modo, vislumbram-se novos paradigmas de cuidado que balizem a concepção científica com olhar holístico, tendo o ser humano como centro de preocupações, ao relacionar-se com o contexto social no qual se encontra inserido, bem como respeitar sua diversidade cultural. "[...] O homem é um animal amarrado a teias de significados que ele mesmo teceu, assume a cultura como sendo essas teias e a sua análise; portanto, não como uma ciência experimental em busca de leis, mas como uma ciência interpretativa, à procura do significado" ${ }^{18: 15}$

Um estudo etnográfico que trata da promoção de saúde e qualidade de vida entre mães de pré-adolescentes em Timbó-SC retrata que: “[...] os enfermeiros têm um compromisso com o cuidado à saúde, promovendo saúde em primeiro lugar, e aumentando a sensibilidade cultural de acordo com os contextos nos quais este estudo vem sendo desenvolvido". 29:1141

Nesta perspectiva, a Enfermagem pode contribuir com o desenvolvimento de habilidades/ atitudes individuais e coletivas da população, re-significando o cuidado, tendo em vista a promoção da saúde.

\section{CONSIDERAÇÕES FINAIS}

A promoção da saúde foi vista neste estudo como possibilidade de aproximar o cuidado de enfermagem com o cuidado que emerge da sabedoria popular no contexto paroara. Isso implica (re)significar a práxis de enfermagem, projetando-a para uma relação sujeito-sujeito, isto é, uma relação de con-vivência e inter-ação "[...] onde experimentamos os seres como sujeitos, como valores, como símbolos que remetem a uma Realidade fontal. [...] o ser humano pode escutar e interpretar esses 
sinais". ${ }^{30: 95}$ Buscou-se o conceito de promoção da saúde com enfoque em uma de suas estratégias, o desenvolvimento de habilidades/atitudes pessoais, discutida a partir da Carta de Otawa. ${ }^{1}$

A Antropologia foi fundamental para melhor apreensão do outro e perceber como esse outro vê a sua necessidade de cuidado. Colocar-se no lugar do outro,buscando compreender para além do seu próprio conhecimento foram fatores que contribuíram para aquilo que aqui chamamos de cuidado de enfermagem paroara. Esse cuidado implica ações que visem compreender a complexidade humana por intermédio de um reconhecimento cultural, isto é, que considere seus hábitos e valores. Assim, no cuidado deve ser valorizada a importância dos princípios, processos dinâmicos, influências do meio ao qual está inserido, bem como o significado de padrões culturais do homem, respeitando sua diversidade e subjetividade, oriundas de seu próprio contex to em interação com o ambiente de suas experiências vivenciadas.

Esse cuidado se reveste de uma concepção que valoriza as condições de vida dos paroaras. Incorporar o cuidado desenvolvido por essa população em seu cotidiano valorizando o uso de ervas, ungüentos, óleos, crenças, vislumbra ultrapassar os discursos já incorporados e pôr em prática as ações educativas de promoção à saúde dos paroaras. As considerações esboçadas neste ensaio configuram-se como preâmbulos - não por isso menos assertivas - de um processo de educação em saúde que tem como eixo central o cuidado na promoção da saúde e suas relações com a cultura do mundo contemporâneo.

Além disso, um dos grandes desafios do cuidado da promoção da saúde, principalmente no contexto latino americano, é a de mudança nas condições de vida, que se caracterizam por um desenvolvimento econômico excludente, relações injustas e condições de vida iníquas. A luta por saúde equivale à melhoria da vida (renda, educação, transporte, lazer, habitação, etc) e deve estar presente nas principais estratégias de promoção, como a do desenvolvimento de habilidades/atitudes que pode ser incorporado no cuidado de enfermagem.

Por outro lado, adotar os princípios da promoção da saúde é um caminho ainda em construção para a melhoria da qualidade de vida que favoreça o desenvolvimento humano. Isto deve envolver solidariedade, interdisciplinaridade, articulação e comunicação entre os atores sociais, para que possam contribuir com a melhoria da qualidade de vida da população.

\section{REFERENCIAS}

1. World Health Organization. The Ottawa Carter for Health Promotion. Ottawa (CA): WHO; 1986.

2. Leavell H; CLARK GG. Medicina Preventiva. Rio de Janeiro (RJ): Editora McGraw-Hill do Brasil; 1978.

3. World Health Organization. The 6th Global Conference on Health Promotion [página da Internet]. 2007 [acesso em 2007 Nov 17]. Disponível em: http://www.who.int/healthpromotion/ conferences/6gchp/en/

4. Heidemann ITS. A promoção da saúde e a concepção dialógica de Freire: possibilidades de sua inserção e limites no processo de trabalho das equipes de saúde da família [tese]. Ribeirão Preto (SP): Universidade de São Paulo. Escola de Enfermagem de Ribeirão Preto; 2006.

5. Labonte R. Centre for Health Promotion. University of Toronto. Toronto (CA): Issues in Health Promotion Series, 1993.

6. Ayres JRCM. Cuidado e reconstrução das práticas de Saúde. Interface Comun Saúde Educ. 2004 Fev; 8(14):73-92.

7. Zagonel IPS. Exercício do poder diante da complexidade das relações no espaço médicohospitalar e de enfermagem. Cogitare Enferm. 1996 Jul-Dez; 1(2):75-80.

8. Silva AL. Cuidado transdimensional: um paradigma emergente. Pelotas (RS): UFPEL; 1997.

9. Souza ML, Sartor VVB, Padilha MICS, Prado ML. O cuidado em enfermagem - uma aproximação teórica. Texto Contexto Enferm. 2005 Abr-Jun; 14(2):266-70.

10. Schaurich D, Crossetti MGO. O elemento dialógico no cuidado de enfermagem: um ensaio com base em Martin Buber. Esc Anna Nery Rev Enferm. 2008 Set; 12(3):544-8.

11. Matta R. A casa e a rua. São Paulo (SP): Brasiliense; 1985.

12. Nordenfelt L. Conversando sobre saúde: um diálogo filosófico. Florianópolis (SC): Bernúncia; 2000.

13. González LJF, Domingos TRE. Antropologia e educação (Cadernos de Antropologia da Educação v. 1). Petrópolis (RJ): Vozes; 2005.

14. Freire P. Educação como prática da liberdade. $17^{\mathrm{a}}$ ed. Rio de Janeiro (RJ): Paz e Terra; 1979.

15. Universidade Federal do Pará. Faculdade de Enfermagem. Novo modelo político- pedagógico. Projeto pedagógico do Curso de Graduação em Enfermagem - UFPA [página da Internet]. 2008 [acesso em 2008 Mai 02]. Disponível em http://www.ufpa.br/ccs/enfermagem/ novoprojetopedagogicodocursodeenfermagem.pdf

16. Ministério da Saúde (BR). Declaração de Alma-Ata. Conferência Internacional sobre cuidados primários de saúde; 6-12 de setembro 1978; Alma-Ata; USSR. In: Ministério da Saúde (BR). Secretaria de Políticas 
de Saúde. Projeto Promoção da Saúde. Declaração de Alma-Ata; Carta de Ottawa; Declaração de Adelaide; Declaração de Sundsvall; Declaração de Santafé de Bogotá; Declaração de Jacarta; Rede de Megapaíses; Declaração do México. Brasília (DF): Ministério da Saúde; 2001. p. 15.

17. Gil TG, Gimeno LM, González RL. Antropología de los cuidados en el ámbito académico de la enfermería en España. Texto Contexto Enferm. 2006 Jan-Mar; 15(1):155-61.

18. Geertz C. A interpretação das culturas. Rio de Janeiro (RJ): LCT-Livros Técnicos e Científicos; 1989.

19. Prado ML, Backes VMS, Santana ME, Souza, MLS. Políticas públicas na formação em saúde: contribuição da enfermagem para superação das desigualdades regionais brasileiras. Texto Contexto Enferm. 2007 Jul-Set; 16(3):531-5.

20. Mapa de Exclusão Social do Estado do Pará [página na internet]. Pará; 2007. [acesso em 2008 Jun 03]. Disponível em: http:/ / www.sepof.pa.gov.br/pdf/ MapaExclusaoSocialPA.pdf

21. Alves PC, Minayo, MCS. Saúde e doença um olhar antropológico, organizadores. Rio de Janeiro (RJ): Fiocruz; 1994.

22. Maués RH. Medicinas populares e "pajelança cabocla" na Amazônia. In: Alves PC, Minayo MCS, organizadores. Saúde e doença: um olhar antropológico. Rio de Janeiro (RJ): Fiocruz; 1998. p.78-81.
23. Oliveira FJA. Concepções de doença: o que os serviços de saúde tem a ver com isso?. In Duarte LF, Leal OF, organizadores. Doença, sofrimento, perturbação: perspectivas etnográficas. Rio de Janeiro (RJ): Fiocruz; 1998. p. 81-94.

24. Ayres JRCM, França Júnior I, Calazans GJ, Saletti Filho HC. O conceito de vulnerabilidade e as práticas de saúde: novas perspectivas e desafios. In: Czeresnia D, Freitas CM, organizadores. Promoção da saúde: conceitos, reflexões, tendências. Rio de Janeiro (RJ): Fiocruz; 2003. p. 117-39.

25. Leininger MM. Caring: an essential human need. Detroid (US): Slack; 1991.

26. George JB. Teorias de enfermagem. Porto Alegre (RS): Artes Médicas, 1993.

27. Boehs AE. Análise dos conceitos de negociação/ acomodação da teoria de M. Leininger. Artigo de revisão. Rev Latino-am Enfermagem. 2002 Jan-Fev; 10(1):90-6.

28. Neves EP. As dimensões do cuidar em enfermagem: concepções teórico-filosóficas. Esc Anna Nery Rev Enferm. 2002 Dez; 6(Supl 1):79-92.

29. Radünz V, Olson J. Promoção de saúde e qualidade de vida entre mães de pré- adolescentes: um estudo etnográfico enfocado em Timbó/SC-Brasil. Rev Latino-am Enfermagem. 2005 Nov-Dez. 13(Esp 2):1135-41.

30. Boff L. Saber cuidar: ética do humano - compaixão pela terra. Petrópolis (SP): Vozes; 1999. 